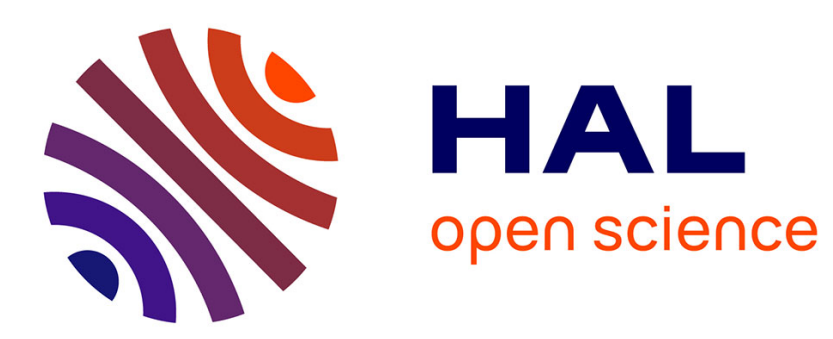

\title{
On the complexity of partitioning a graph into a few connected subgraphs
}

\author{
Julien Bensmail
}

\section{To cite this version:}

Julien Bensmail. On the complexity of partitioning a graph into a few connected subgraphs. Journal of Combinatorial Optimization, 2014, A paraître, http://link.springer.com/journal/10878. hal$00762612 \mathrm{v} 2$

\section{HAL Id: hal-00762612 \\ https://hal.science/hal-00762612v2}

Submitted on 18 Apr 2013

HAL is a multi-disciplinary open access archive for the deposit and dissemination of scientific research documents, whether they are published or not. The documents may come from teaching and research institutions in France or abroad, or from public or private research centers.
L'archive ouverte pluridisciplinaire HAL, est destinée au dépôt et à la diffusion de documents scientifiques de niveau recherche, publiés ou non, émanant des établissements d'enseignement et de recherche français ou étrangers, des laboratoires publics ou privés. 


\title{
On the complexity of partitioning a graph into a few connected subgraphs
}

\author{
Julien Bensmail
}

the date of receipt and acceptance should be inserted later

\begin{abstract}
Given a graph $G$, a sequence $\tau=\left(n_{1}, \ldots, n_{p}\right)$ of positive integers summing up to $|V(G)|$ is said to be realizable in $G$ if there exists a realization of $\tau$ in $G$, i.e. a partition $\left(V_{1}, \ldots, V_{p}\right)$ of $V(G)$ such that each $V_{i}$ induces a connected subgraph of $G$ on $n_{i}$ vertices. We first give a reduction showing that the problem of deciding whether a sequence with $c$ elements is realizable in a graph is NP-complete for every fixed $c \geq 2$. Thanks to slight modifications of this reduction, we then prove additional hardness results on decision problems derived from the previous one. In particular, we show that the previous problem remains NP-complete when a constant number of vertex-membership constraints must be satisfied. We then prove the tightness of an easiness result proved independently by Györi and Lovász regarding a similar problem. We finally show that another graph partition problem, asking whether several partial realizations of $\tau$ in $G$ can be extended to obtain whole realizations of $\tau$ in $G$, is $\Pi_{2}^{p}$-complete.
\end{abstract}

Keywords arbitrarily partitionable graphs · partition into connected subgraphs $\cdot$ partition under vertex prescriptions $\cdot$ complexity $\cdot$ polynomial hierarchy

\section{Introduction}

Let $G$ be a connected graph. A sequence $\tau=\left(n_{1}, \ldots, n_{p}\right)$ of positive integers is admissible for $G$ if $\sum_{i=1}^{p} n_{i}=|V(G)|$. We say that $\tau$ is realizable in $G$ if $\tau$ is admissible for $G$ and there exists a realization of $\tau$ in $G$, i.e. a partition $\left(V_{1}, \ldots, V_{p}\right)$ of $V(G)$ such that $V_{i}$ induces a connected subgraph of $G$ on $n_{i}$

J. Bensmail

Univ. Bordeaux, LaBRI, UMR 5800, F-33400 Talence, France

CNRS, LaBRI, UMR 5800, F-33400 Talence, France

E-mail: julien.bensmail@labri.fr

Tel.: +33-(0)5-40-00-35-17, Fax: +33-(0)5-40-00-66-69 
vertices for every $i \in\{1, \ldots, p\}$. We refer to $p$ as the size of $\tau$. A $p$-sequence is a sequence with size $p$.

The problem of finding a realization of a given sequence in a graph gained a lot of attention since the result, proved independently by Györi and Lovász, which states that, for any $q \geq 1$, every sequence with size at most $q$ admissible for a $q$-connected graph $G$ is realizable in $G[6,7]$. Since then, several graph properties based on the definition above have been investigated.

For instance, we say that $G$ is arbitrarily partitionable (AP for short) if every sequence admissible for $G$ is also realizable in $G$. For the sake of the upcoming next definitions, let us now consider a $k$-prescription of $G$, i.e. a sequence $\left(v_{1}, \ldots, v_{k}\right)$ of $k$ pairwise distinct vertices of $G$ with $k \leq p$, where $p$ is the size of a sequence $\tau$ admissible for $G$. We say that $\tau$ is realizable in $G$ under $\left(v_{1}, \ldots, v_{k}\right)$ if there exists a realization $\left(V_{1}, \ldots, V_{p}\right)$ of $\tau$ in $G$ such that $v_{i} \in V_{i}$ for every $i \in\{1, \ldots, k\}$. In other words, a $k$-prescription is a set of vertices that were chosen to belong to the first $k$ parts of a realization of $\tau$ in $G$. Notice that, in our terminology, the $k$ part sizes associated with these $k$ prescribed vertices are the first $k$ ones of the sequence. Finally, the graph $G$ is said to be arbitrarily partitionable under $k$ prescriptions (AP $+k$ for short) if every sequence with size at least $k$ admissible for $G$ is realizable in $G$ under every $k$-prescription of $G$. All these definitions were introduced to deal with a practical problem of resource sharing among an arbitrary number of users $[1$, 3].

In this paper, we consider the computational complexity of some decision problems derived from the definitions above. Thanks to our main reduction given in Section 2, we show that the problem of deciding whether a sequence is realizable in a given graph is $\mathrm{NP}$-complete even when restricted to $c$-sequences for every fixed $c \geq 2$. This reduction may be related to one reduction from [5], where similar gadgets as ours are used to prove the hardness of a min-max tree partition problem. We then augment our reduction in further sections to show additional complexity results. We first prove in Section 3 that requesting prescriptions while partitioning a graph does not alter the complexity of the problem, and this no matter how many such prescriptions are requested. In Section 4, we investigate the tightness of the well-known easiness result proved independently by Györi and Lovász mentioned above. We finally discuss the complexity of the problems of deciding whether a graph is $\mathrm{AP}$ or $\mathrm{AP}+k$ in Section 5. We locate these two problems in the $\Pi_{2}^{p}$ complexity class of the polynomial hierarchy and explain why we cannot modify our previous reductions to prove that these problems are $\Pi_{2}^{p}$-complete. We however show that our graph partition problem is not "incompatible" with the notion of $\Pi_{2}^{p}$-complete problems by pointing out one such $\Pi_{2}^{p}$-complete problem.

\section{Complexity of partitioning a graph into a few connected subgraphs}

In this section, we focus on the following decision problem. 
Realizable Sequence - RealSeq

Instance: A graph $G$ and a sequence $\tau$.

Question: Is $\tau$ realizable in $G$ ?

Assuming that the size of $\tau$ is constant, we get the following refinement.

ReAlizable $k$-SEQuence - $k$-ReALSEQ

Instance: A graph $G$ and a $k$-sequence $\tau$.

Question: Is $\tau$ realizable in $G$ ?

It is already known that REALSEQ is computationally hard, even under restrictions on $G$ or $\tau$. In particular, this problem remains NP-complete even when $G$ is a tree with maximum degree 3 , or $\tau=(k, \ldots, k)$ has only one integer value $k \geq 3$ that divides $|V(G)|$ (see [2] and [4], respectively). However, the complexity reductions used to show these restrictions on REALSEQ do not imply the existence of a constant threshold $c \geq 1$ such that:

$-k$-REALSEQ is in P for every $k \leq c-1$;

$-k$-REALSEQ is NP-complete otherwise.

The answer to an instance of 1-REALSEQ is yes if and only if $G$ is connected. Since the connectedness of a graph can be checked easily, we have $c \geq 2$ assuming that $c$ exists. In what follows, we prove that $c=2$, i.e. that $k$ REALSEQ is NP-complete for every $k \geq 2$. Our reduction is from the following variant of $3 \mathrm{SAT}$.

\section{1-IN-3 SAT}

Instance: A $3 \mathrm{CNF}$ formula $F$ over variables $\left\{x_{1}, \ldots, x_{n}\right\}$ and clauses $\left\{C_{1}, \ldots, C_{m}\right\}$. Question: Is there a 1-in-3 truth assignment of the variables of $F$, i.e. a truth assignment such that each clause of $F$ has exactly one true literal?

Notice that we can suppose that every possible literal appears in $F$. Indeed, if $x_{i}$ does not appear in any clause of $F$, then the 3CNF formula $F^{\prime}=F \wedge$ $\left(x_{i} \vee \overline{x_{i}} \vee x_{n+1}\right) \wedge\left(x_{n+1} \vee x_{n+1} \vee \overline{x_{n+1}}\right)$, where $x_{n+1}$ is a new variable, admits a 1-in-3 truth assignment of its variables if and only if $F$ admits one too. Since there are $2 n$ literals related to the variables of $F$, a formula equivalent to $F$ that contains every possible literal over its variables can be obtained from $F$ in polynomial time.

Our proof of the NP-completeness of $k$-REALSEQ for every $k \geq 2$ reads as follows. We first show in Theorem 1 below that 2-REALSEQ is NP-complete by reduction from 1-IN-3 SAT. We then explain, in Theorem 2, how to modify our reduction from 1-IN-3 SAT to 2-REALSEQ so that we get a reduction from $1-\mathrm{IN}-3$ SAT to $k$-REALSEQ for any $k \geq 3$.

Theorem 1 2-REALSEQ is NP-complete.

Proof First of all, ReALSEQ is clearly in NP. One can indeed design an algorithm that takes the graph $G$, the sequence $\tau$ and a realization $R$ of $\tau$ in $G$ as input and checks whether $R$ is correct. More precisely, such an algorithm has to check that $\tau$ is admissible for $G$, the parts of $R$ have the correct sizes 


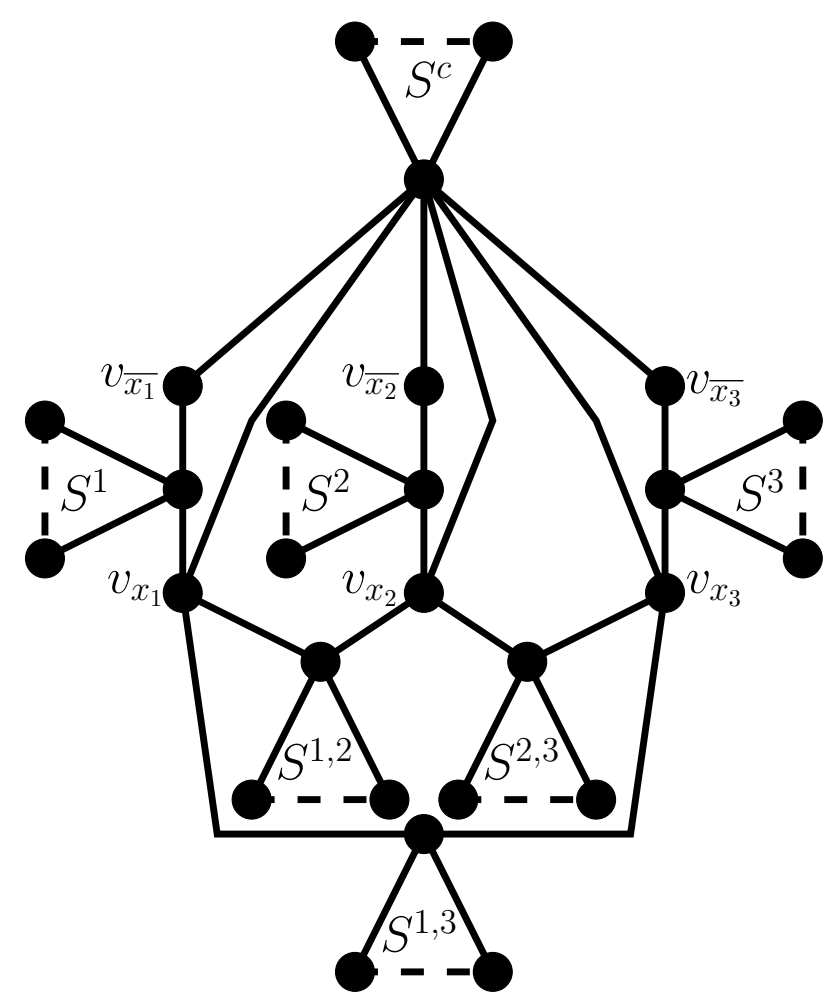

Fig. 1 Resulting subgraph in the clause subgraph of $G_{F}$ for a clause $C_{1}=\left(x_{1} \vee x_{2} \vee x_{3}\right)$ of $F$

regarding $\tau$, and that the subgraphs of $G$ induced by $R$ are connected. This verification can be done in polynomial time regardless of the size of $\tau$.

We now prove that 2-REALSEQ is NP-complete by reduction from 1-IN-3 SAT. For a given formula $F$ over variables $\left\{x_{1}, \ldots, x_{n}\right\}$ and clauses $\left\{C_{1}, \ldots, C_{m}\right\}$, we construct a graph $G_{F}$ and a sequence $\tau_{F}$ admissible for $G_{F}$ such that $F$ is satisfiable in a 1-in-3 way if and only if $\tau_{F}$ is realizable in $G_{F}$. Our reduction is performed in such a way that $\tau_{F}$ is a 2 -sequence.

The graph $G_{F}$ is composed of two main vertex-disjoint subgraphs. The first one is the clause subgraph. Each literal $\ell_{i}$ of $F$ is associated with a literal vertex $v_{\ell_{i}}$ in the clause subgraph. For each pair of literals $\ell_{i}$ and $\overline{\ell_{i}}$ of $F$, we then link the literal vertices $v_{\ell_{i}}$ and $v_{\overline{\ell_{i}}}$ to the root vertex of a star $S^{i}$ with $n$ vertices of degree 1 . Two literal vertices $v_{\ell_{i}}$ and $v_{\ell_{j}}$ such that $\ell_{j} \neq \overline{\ell_{i}}$ are similarly linked to the root vertex of a star $S^{i, j}$ with $n$ vertices of degree 1 if they both appear in a same clause of $F$. We finally add a control star $S^{c}$ with $n$ vertices of degree 1 to the clause subgraph of $G_{F}$ and link its root to every literal vertex so that the clause subgraph is connected.

The construction so far is detailed in Figure 1. Let $n_{2}$ be the number of vertices of the clause subgraph. Then we have 


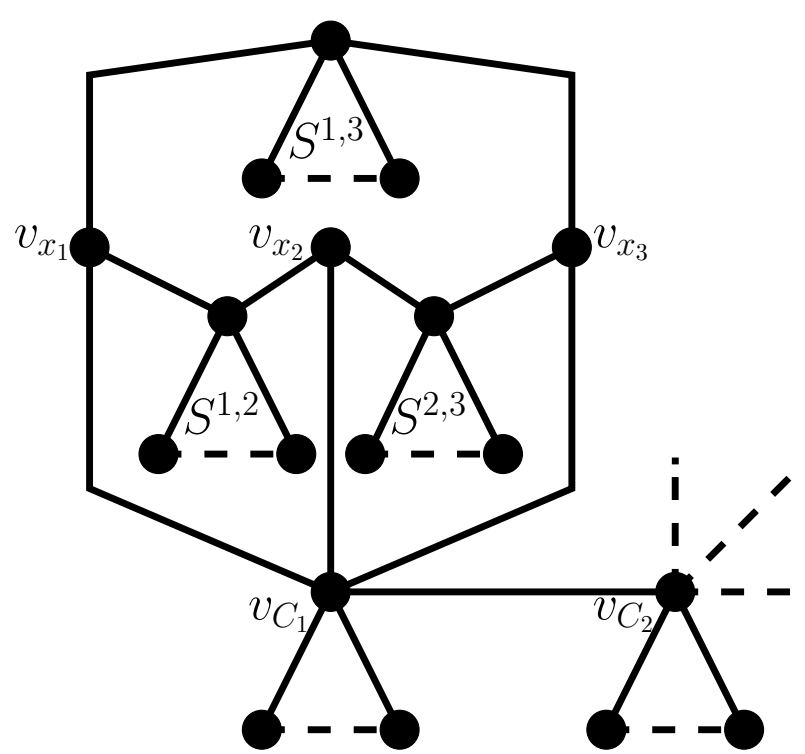

Fig. 2 Connection between the base and clause subgraphs of $G_{F}$ for a clause $C_{1}=\left(x_{1} \vee\right.$ $\left.x_{2} \vee x_{3}\right)$ of $F$

$$
n_{2} \leq 2 n+n(n+1)+3 m(n+1)+n+1
$$

since there are exactly $2 n$ literals and $n$ pairs of literals of the form $\left\{\ell_{i}, \overline{\ell_{i}}\right\}$ in $F$, all the clauses of $F$ can have distinct literals, and the control star $S^{c}$ has exactly $n$ vertices of degree 1 .

The second subgraph of $G_{F}$ is the base subgraph. With each clause $C_{i}$ in $F$ we associate a clause vertex $v_{C_{i}}$ in the base subgraph that is linked to $n_{2}-n$ vertices of degree 1 . For each $i \in\{1, \ldots, m-1\}$, we finally add the edge $v_{C_{i}} v_{C_{i+1}}$ to $E\left(G_{F}\right)$ so that the clause vertices induce a path in $G_{F}$. If we denote by $n_{1}$ the number of vertices of the base subgraph of $G_{F}$, then we have

$$
n_{1}=m\left(n_{2}-n+1\right) \text {. }
$$

We end up the construction of $G_{F}$ by adding some edges between the base and clause subgraphs of $G_{F}$ : for each clause $C_{i}=\left(\ell_{i_{1}} \vee \ell_{i_{2}} \vee \ell_{i_{3}}\right)$ in $F$, we add $v_{C_{i}} v_{\ell_{i_{1}}}, v_{C_{i}} v_{\ell_{i_{2}}}$ and $v_{C_{i}} v_{\ell_{i_{3}}}$ to $E\left(G_{F}\right)$. See Figure 2 for an illustration of this connection.

The number of vertices of $G_{F}$ is $n_{1}+n_{2}$. Thus, the construction of $G_{F}$ is performed in polynomial time regarding the size of $F$. Consider now the sequence $\tau_{F}=\left(n_{1}+n, n_{2}-n\right)$. Since the two elements of $\tau_{F}$ are strictly greater than 1 , any part $U$ from a realization $R$ of $\tau_{F}$ in $G_{F}$ that covers the root vertex of any star subgraph in $G_{F}$ must also contain all the vertices of degree 1 attached to it. Indeed, if this were not the case, then the graph $G_{F}-U$ would contain at least two connected components and, thus, the part of $R$ different from $U$ could not induce a connected subgraph of $G_{F}$. 
For this reason, observe that, because of all the induced stars $S_{n_{2}-n+1}$ in the base subgraph of $G_{F}$, this subgraph must be covered by the part $V_{1}$ with size $n_{1}+n$ of a realization $\left(V_{1}, V_{2}\right)$ of $\tau_{F}$ in $G_{F}$. Starting from this, we then have to add $n$ additional vertices from the clause subgraph of $G_{F}$ to $V_{1}$. For a similar reason as the one above, we can only pick up some literal vertices of $G_{F}$ since picking up any other of its vertices would disconnect $G_{F}$ into too many small components. According to our construction, we cannot also add to $V_{1}$ two literal vertices $v_{\ell_{i}}$ and $v_{\ell_{j}}$ such that $\ell_{i}$ and $\ell_{j}$ are a variable of $F$ and its negation, or appear in a same clause of $F$, since otherwise this would once again make the subgraph $G_{F}-V_{1}$ disconnected.

We can then deduce a 1-in-3 truth assignment of the variables of $F$ from a realization $R=\left(V_{1}, V_{2}\right)$ of $\tau_{F}$ in $G_{F}$ and vice-versa. If $R$ is a correct realization of $\tau_{F}$ in $G_{F}$, then there are exactly $n$ literal vertices $v_{\ell_{i_{1}}}, \ldots, v_{\ell_{i_{n}}}$ from the clause subgraph of $G_{F}$ that belong to $V_{1}$. Since $G_{F}\left[V_{2}\right]$ is connected, setting the literals $\ell_{i_{1}}, \ldots, \ell_{i_{n}}$ true makes $F$ evaluated true in a 1 -in-3 way since no pair of these literals is a variable of $F$ and its negation or appears in a same clause of $F$. Conversely, if $F$ is satisfiable in a 1 -in-3 way, then let $\phi:\left\{\ell_{1}, \ldots, \ell_{2 n}\right\} \rightarrow$ $\{0,1\}$ be a satisfying 1 -in- 3 truth assignment of its literals. Then observe that $\left(V_{1}, V_{2}\right)$, where

- $V_{1}$ contains all the vertices from the base subgraph of $G_{F}$ and every literal vertex $v_{\ell_{i}}$ from the clause subgraph of $G_{F}$ such that $\phi\left(\ell_{i}\right)=1$,

$-V_{2}=V\left(G_{F}\right)-V_{1}$

is a correct realization of $\tau_{F}$ in $G_{F}$ according to the arguments above.

We finally explain how to generalize the reduction of Theorem 1 so that we get a reduction from 1 -IN-3 SAT to $k$-REALSEQ for any $k \geq 3$.

Theorem $2 k$-REALSEQ is NP-complete for every $k \geq 2$.

Proof $k$-REALSEQ is in NP for every $k \geq 2$ as mentioned in the proof of Theorem 1 . The proof that $k$-REALSEQ is NP-complete for every $k \geq 3$ is based on our reduction from 1-IN-3 SAT to 2-REALSEQ. More precisely, we modify the instance resulting from the reduction, i.e. the graph $G_{F}$ and the sequence $\tau_{F}$, in such a way that the arguments given in the proof of Theorem 1 are still correct and not altered by the modifications.

For the sake of this proof, let us introduce the following definition. Given a graph $H$, a vertex $v \in V(H)$ and an arbitrary integer $a \geq 3$, the $(a, v)$-staraugmentation of $H$ is the graph obtained as follows:

1. consider the union of $H$ and a star $S^{a}$ with $a-1$ vertices of degree 1 ,

2. add an edge between $v$ and the root of $S^{a}$.

An example of an $(a, v)$-star-augmentation of a graph is depicted in Figure 3. Let us first show that 3-REALSEQ is NP-complete by reduction from 1-IN-3 SAT before generalizing our arguments. From a $3 \mathrm{CNF}$ formula $F$, we construct a graph $G_{F}$ and a sequence $\tau_{F}=\left(n_{1}, n_{2}, n_{3}\right)$ admissible for $G_{F}$ such that $F$ is satisfiable in a 1 -in-3 way if and only if $\tau_{F}$ is realizable in $G_{F}$. 

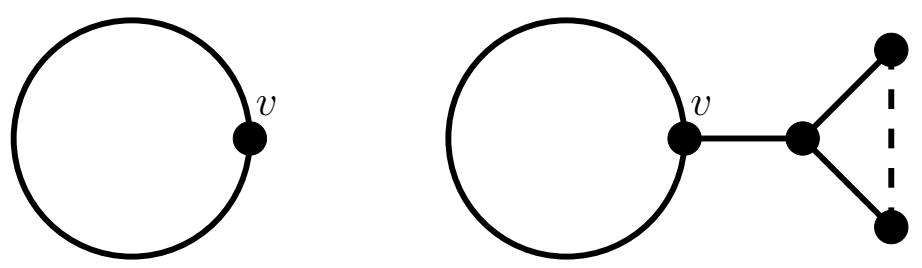

Fig. 3 A graph $H$ and an arbitrary $(a, v)$-star-augmentation of $H$

By applying the reduction from 1-IN-3 SAT to 2-REALSEQ, we get a graph $G_{F}^{\prime}$ and a sequence $\tau_{F}^{\prime}=\left(n_{1}^{\prime}, n_{2}^{\prime}\right)$ admissible for $G_{F}^{\prime}$ that is realizable in $G_{F}^{\prime}$ if and only if $F$ admits a 1-in-3 assignment of its variables. Besides, recall that $n_{1}^{\prime}, n_{2}^{\prime} \geq 2$. Now consider, as $G_{F}$, an $(a, v)$-star-augmentation of $G_{F}^{\prime}$ where $a=n_{1}^{\prime}+n_{2}^{\prime}+1$ and $v \in V\left(G_{F}^{\prime}\right)$ is arbitrary, and $\tau_{F}=\left(a, n_{1}^{\prime}, n_{2}^{\prime}\right)$. In a realization $\left(U, V_{1}, V_{2}\right)$ of $\tau_{F}$ in $G_{F}$, notice that, because $n_{1}^{\prime}, n_{2}^{\prime} \geq 2$, the star subgraph $S^{a}$ of $G_{F}$ resulting from the star augmentation must be covered entirely by the part $U$ with size $a$ since covering it with one of the other two parts would disconnect $G_{F}$ into too many small components. Therefore, $\tau_{F}$ is realizable in $G_{F}$ if and only if $\tau_{F}^{\prime}$ is realizable in $G_{F}^{\prime}$, and by transitivity we get that $F$ is satisfiable in a 1 -in-3 way if and only if $\tau_{F}$ is realizable in $G_{F}$.

One can repeat the previous procedure as many times as wanted until $\tau_{F}$ has the requested size. At each step, we get another instance of REALSEQ which is equivalent to the previous one but whose sequence has one more element. Said differently, from the instance $F$ of 1 -IN-3 SAT we first construct an equivalent instance of 2-REALSEQ. From this instance of 2-REALSEQ is then obtained an equivalent instance of 3-REALSEQ thanks to a star-augmentation. With the same construction, we then get an equivalent instance of 4-REALSEQ. And so on. All these reduced instances are obtained in polynomial time, and are equivalent to $F$ by transitivity. We thus get that $k$-REALSEQ is NPcomplete for every $k \geq 3$.

\section{Complexity of partitioning a graph into connected subgraphs following a prescription}

In this section, we investigate the computational complexity of the following decision problem.

Realizable Sequence Under Prescriptions - PrescSeq

Instance: A graph $G$, a sequence $\tau$, and a prescription $P$ of $G$. Question: Is $\tau$ realizable in $G$ under $P$ ?

Similarly as for REALSEQ and its refinement $k$-REALSEQ, we introduce a refined version of PRESCSEQ obtained by assuming that the sizes of $\tau$ and $P$ are fixed. 

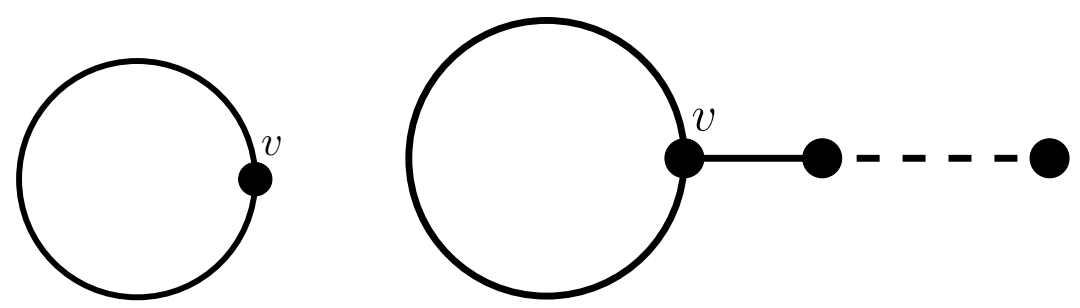

Fig. $4 \mathrm{~A}$ graph $H$ and an arbitrary $(a, v)$-path-augmentation of $H$

Realizable $k$-Sequence Under $k^{\prime}$ Prescriptions - $\left(k, k^{\prime}\right)$-PrescSeq

Instance: A graph $G$, a $k$-sequence $\tau$, and a $k^{\prime}$-prescription $P$ of $G$.

Question: Is $\tau$ realizable in $G$ under $P$ ?

For any problem $\left(k, k^{\prime}\right)$-PrescSeQ, we have $k \geq k^{\prime}$ by definition. Besides, $(k, 0)$-PrescSeQ is equivalent to $k$-RealSeQ which was shown to be in $\mathrm{P}$ when $k=1$, and NP-complete for every $k \geq 2$ (Section 2). Note further that the answer to an instance of $(1,1)$-PrescSeQ is yes if and only if $G$ is connected. Therefore, $(1,1)$-PrescSeQ is in P.

We now prove that the remaining problems $\left(k, k^{\prime}\right)$-PRESCSEQ, i.e. with $k \geq 2$ and $1 \leq k^{\prime} \leq k$, are NP-complete.

Theorem $3\left(k, k^{\prime}\right)$-PRESCSEQ is NP-complete whenever $k \geq 2$.

Proof One can clearly modify the checking algorithm for REALSEQ we gave in the proof of Theorem 1 so that it also takes the prescription $P$ as an input and makes sure that the vertices of $P$ belong to the corresponding parts of $R$. This modification does not alter the complexity of the algorithm. Therefore, PrescSeQ is in NP.

Let $k$ and $k^{\prime}$ be fixed. Clearly, if $k^{\prime}=0$, then $\left(k, k^{\prime}\right)$-PRESCSEQ is NPcomplete by Theorem 2. Suppose thus that $k^{\prime} \geq 1$. We show that $\left(k, k^{\prime}\right)$ PRESCSEQ is NP-complete thanks to our reduction from 1-IN-3 SAT to $k$ REALSEQ (Theorem 2) and the following construction. Let $a \geq 1$ be an arbitrary positive integer and $v$ be an arbitrary vertex of some graph $H$. The $(a, v)$-path-augmentation of $H$ is the graph obtained from $H$ as follows:

1. consider the union of $H$ and $P_{a}$, a path of order $a$ whose vertices are consecutively denoted by $u_{1}, \ldots, u_{a}$;

2. add an edge between $u_{1}$ and $v$.

This construction is depicted in Figure 4. First suppose that $k-k^{\prime} \geq 2$. From $F$, start by constructing a graph $G_{F}$ and a sequence $\tau_{F}=\left(n_{1}, \ldots, n_{k-k^{\prime}}\right)$ with size $k-k^{\prime}$ admissible for $G_{F}$ such that $F$ is satisfiable in a 1-in-3 way if and only if $\tau_{F}$ is realizable in $G_{F}$. This graph $G_{F}$ and sequence $\tau_{F}$ may be obtained thanks to the reduction from Theorem 2 since $k-k^{\prime} \geq 2$. Let us now denote by $G_{F}^{\prime}$ the graph obtained from $G_{F}$ by performing $k^{\prime}$ arbitrary path-augmentations, e.g. one $\left(a_{1}, v\right)$-path-augmentation, one $\left(a_{2}, v\right)$ path-augmentation, etc., for some $v \in V\left(G_{F}\right)$ and integers $a_{1}, \ldots, a_{k^{\prime}} \geq 1$. Let 
$u_{1}, \ldots, u_{k^{\prime}}$ denote the vertices with degree 1 of the resulting hanging paths, where $u_{i}$ is the endvertex of the $i^{t h}$ path-augmentation. Finally, let $\tau_{F}^{\prime}=$ $\left(a_{1}, \ldots, a_{k^{\prime}}, n_{1}, \ldots, n_{k-k^{\prime}}\right)$ and $P_{F}^{\prime}=\left(u_{1}, \ldots, u_{k^{\prime}}\right)$ be a $k$-sequence admissible for $G_{F}^{\prime}$ and a $k^{\prime}$-prescription of $G_{F}^{\prime}$, respectively.

Since the first $k^{\prime}$ parts $U_{1}, \ldots, U_{k^{\prime}}$ of a realization of $\tau_{F}^{\prime}$ in $G_{F}^{\prime}$ under $P_{F}^{\prime}$ must induce connected subgraphs of $G_{F}^{\prime}$ on $a_{1}, \ldots, a_{k^{\prime}}$ vertices, respectively, including $u_{1}, \ldots, u_{k^{\prime}}$, respectively, the only way for choosing the part $U_{i}$ is to pick up every vertex resulting from the $i^{\text {th }}$ path-augmentation. Once these parts have been picked up, we still have to find a realization $\left(V_{1}, \ldots, V_{k-k^{\prime}}\right)$ of the remaining sequence $\left(n_{1}, \ldots, n_{k-k^{\prime}}\right)=\tau_{F}$ in the remaining graph $G_{F}^{\prime}-\bigcup_{i=1}^{k^{\prime}} U_{i}=G_{F}$. Hence, $\tau_{F}^{\prime}$ is realizable in $G_{F}^{\prime}$ under $P_{F}^{\prime}$ if and only if $\tau_{F}$ is realizable in $G_{F}$. By transitivity, we get that $F$ is satisfiable in a 1-in-3 way if and only if $\tau_{F}^{\prime}$ is realizable in $G_{F}^{\prime}$ under $P_{F}^{\prime}$. This reduction can clearly be performed in polynomial time.

Note that this reduction does not work when $k-k^{\prime} \in\{0,1\}$ since, in this situation, too much prescribed vertices are requested. But recall that, in the reduction from 1-IN-3 SAT to 2-REALSEQ, some vertices from the base and clause subgraphs of $G_{F}$, respectively, have to be covered by the parts with size $n_{1}$ and $n_{2}$, respectively, of a realization of $\tau_{F}$ in $G_{F}$. Thus, we could request up to 2 prescriptions, and directly get that $(2,1)$ - and $(2,2)$-PRESCSEQ are NP-complete. By performing the same reduction scheme as above but from one of these two problems, we get that $\left(k, k^{\prime}\right)$-PRESCSEQ is also NP-complete when $k-k^{\prime} \in\{0,1\}$.

\section{Complexity of partitioning a graph with given connectivity into connected subgraphs following a prescription}

In the introduction section, we mentioned a famous result proved independently by Györi and Lovász on the problem of realizing sequences in $q$-connected graphs. Using our terminology, this result may be formulated as follows $[6,7]$.

Theorem 4 (Györi and Lovász, independently) Every sequence with size $k \leq q$ admissible for a $q$-connected graph $G$ is realizable in $G$ under $k$ prescriptions.

Theorem 4 implies that the answer to every instance of $(k, k)$-PRESCSEQ such that $G$ is a $q$-connected graph and $\tau$ is admissible for $G$ is yes whenever $k \leq q$. We now show that this easiness result is in some sense tight, i.e. that prescribing strictly more than $q$ vertices while partitioning a $q$-connected graph is difficult in general.

Theorem $5\left(k, k^{\prime}\right)$-PRESCSEQ is NP-complete when restricted to $q$-connected graphs for every $q \geq 1$ whenever $q<k^{\prime} \leq k$.

Proof The NP part of the claim derives from the NP part of PRESCSEQ which was shown in the proof of Theorem 3 . The hardness part can be proved thanks to our previous reductions from 1-IN-3 SAT. 
First, because $\left(k, k^{\prime}\right)$-PRESCSEQ is NP-complete for every $k \geq 2$ and our proof of this statement was obtained by reducing instances of 1-IN-3 SAT to 1-connected graphs (see Theorem 3), the statement holds for $q=1$.

We now prove the general case, i.e. $q \geq 2$. Let $k^{\prime}>q$ and $k \geq k^{\prime}$ be fixed. Start from a $3 \mathrm{CNF}$ formula $F$, and produce a graph $G_{F}$, a sequence $\tau_{F}=\left(n_{1}, \ldots, n_{k-q+1}\right)$ admissible for $G_{F}$, and a $\left(k^{\prime}-q+1\right)$-prescription $\left(u_{1}, \ldots, u_{k^{\prime}-q+1}\right) P_{F}$ of $G_{F}$ such that $F$ is 1 -in-3 satisfiable if and only if $\tau_{F}$ is realizable in $G_{F}$ under $P_{F}$. This reduced instance may be obtained thanks to the reduction given in the proof of Theorem 1, and the star- and pathaugmentation constructions introduced in the proofs of Theorems 2 and 3 , respectively. Now consider the following instance of $\left(k, k^{\prime}\right)$-PRESCSEQ.

- $G_{F}^{\prime}$ is obtained by successively adding $q-1$ universal vertices $v_{1}, \ldots, v_{q-1}$ to $G_{F}$, i.e. vertices joined to all other vertices of the graph.

$-\tau_{F}^{\prime}=\left(1, \ldots, 1, n_{1}, \ldots, n_{k-q+1}\right)$ is a sequence admissible for $G_{F}^{\prime}$ with $q-11$ 's.

- $P_{F}^{\prime}=\left(v_{1}, \ldots, v_{q-1}, u_{1}, \ldots, u_{k^{\prime}-q+1}\right)$ is a prescription of $G_{F}^{\prime}$.

Clearly, $G_{F}^{\prime}$ is $q$-connected since $G_{F}$ is 1 -connected, and $\tau_{F}^{\prime}$ and $P_{F}^{\prime}$ have size $k$ and $k^{\prime}$, respectively. Besides, since prescribing a vertex to a part with size 1 is like removing it from the graph, what is left once the vertices $v_{1}, \ldots, v_{q-1}$ have been assigned to parts with size 1 of a realization is $G_{F}, \tau_{F}$ and $P_{F}$. Therefore, $\tau_{F}^{\prime}$ is realizable in $G_{F}^{\prime}$ under $P_{F}^{\prime}$ if and only if $\tau_{F}$ is realizable in $G_{F}$ under $P_{F}$. By transitivity, we get that $F$ is 1-in-3 satisfiable if and only if $\tau_{F}^{\prime}$ is realizable in $G_{F}^{\prime}$ under $P_{F}^{\prime}$.

\section{Some $\Pi_{2}^{p}$ problems}

In this section, we investigate the relationship between some graph partition problems derived from our definitions and the $\Pi_{2}^{p}$ complexity class. We start with the following problem.

\section{AP GRAPH}

Instance: A graph $G$.

Question: Is $G$ an AP graph?

This problem is not known to belong to either NP or co-NP. However, it is clearly in $\Pi_{2}^{p}$ since one can design a polynomial-time algorithm that takes $G$ and a sequence $\tau$ admissible for $G$ as input and checks that $\tau$ is not realizable in $G$ using an oracle for REALSEQ.

Consider next the following problem.

\section{$\mathrm{AP}+k$ GRAPH}

Instance: A graph $G$.

Question: Is $G$ an $\mathrm{AP}+k$ graph?

Clearly, $\mathrm{AP}+k$ GrAPH is also in $\Pi_{2}^{p}$ for every $k \geq 1$. Indeed, recall that PRESCSEQ is NP-complete whatever is the number of prescribed vertices (Theorem 3). One can thus design a similar algorithm as the one we just mentioned for AP GRAPH, except that this algorithm uses an oracle for PRESCSEQ. 

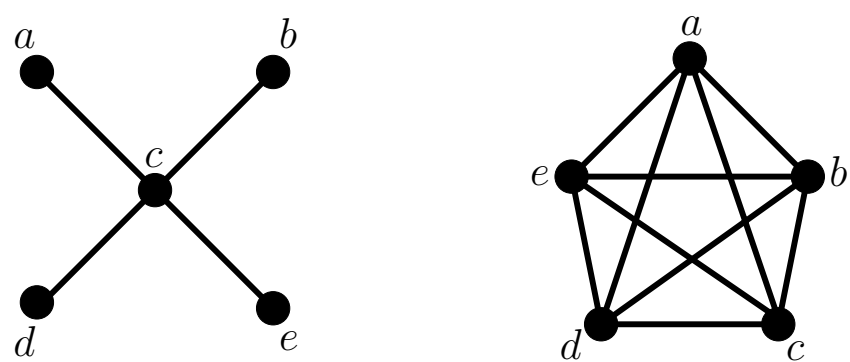

Fig. 5 The graphs $K_{1,4}$ and $K_{5}$

We do not know whether AP GRAPH and AP $+k$ GRAPH are $\Pi_{2}^{p}$-complete problems. Indeed, to design a polynomial-time reduction from a $\Pi_{2}^{p}$-complete problem $A$ to one of these two problems, it would be necessary to "translate" the restrictions associated with an instance of $A$ to some graph substructures just like we did in the proof of Theorem 1 by introducing a lot of star subgraphs in the reduced graphs. But introducing these graph substructures generally makes the whole graph being not AP. That is why, for example, our reduction from 1-IN-3 SAT to REALSEQ does not seem to be generalizable into some reduction from a $\Pi_{2}^{p}$-complete version of 1 -IN-3 SAT to AP GRAPH.

Most of $\Pi_{2}^{p}$ problems are of the form "For every $X$, is there a $Y$ such that...?". The AP GRAPH and AP $+k$ GRAPH problems clearly catch this form since one could reformulate them as "For every admissible sequence, is there a realization such that...?". However, in most of $\Pi_{2}^{p}$-complete problems, the two input objects $X$ and $Y$ have the same nature (e.g. truth assignments, sets of vertices, etc.) while it is not the case for AP GRAPH and AP $+k$ GRAPH. This is another reason why it seems difficult to design a reduction from one classical $\Pi_{2}^{p}$-complete problem to one of these two problems.

In order to show that graph partition problems are not "incompatible" with the notion of $\Pi_{2}^{p}$-complete problems, we introduce another problem. Let $G$ be a graph and $\tau=\left(n_{1}, \ldots, n_{p}\right)$ be a sequence admissible for $G$. Given a $\ell \in\{1, \ldots, p\}$, a $n_{\ell}$-partition-level for $\tau$ and $G$ is a set $L_{\ell}$ of subsets of $V(G)$ that induce connected subgraphs of $G$ with order $n_{\ell}$. A $\left(n_{1}, \ldots, n_{\ell}\right)$-partitionhierarchy $L$ for $\tau$ and $G$ is a collection $L=\left(L_{1}, \ldots, L_{\ell}\right)$ of $n_{i}$-partition-levels for $\tau$ and $G$ for $i$ up to $\ell$ such that no subsets in $L_{i}$ and $L_{j}$ intersect for $i \neq j$. We finally say that $\tau$ is realizable in $G$ under $L$ if for every collection of subsets $\left(V_{1}, \ldots, V_{\ell}\right)$ from the partition levels of $L$ such that $V_{1} \in L_{1}, \ldots, V_{\ell} \in L_{\ell}$ there exists a realization $\left(V_{1}, \ldots, V_{\ell}, \ldots, V_{p}\right)$ of $\tau$ in $G$. In other words, we are given partial realizations of $\tau$ in $G$, i.e. some ways for picking up the parts associated with the $\ell$ first elements of $\tau$, whose parts are dispatched into $\ell$ partition levels, and we ask whether each of these partial realizations is extendable to a whole realization of $\tau$ in $G$. A partition hierarchy is actually a compact way to describe a large number of partial realizations.

As an illustration of these definitions, consider the two graphs $K_{1,4}$ and $K_{5}$ of Figure 5 . Let $\tau=(1,1,3)$ be a sequence admissible for $K_{1,4}$ and $K_{5}$, 
let $L_{1}=(\{a\},\{c\})$ and $L_{2}=(\{b\},\{e\})$ be two 1-partition-levels for $\tau$ and both $K_{1,4}$ and $K_{5}$, and $L=\left(L_{1}, L_{2}\right)$ be a $(1,1)$-partition-hierarchy for $\tau$ and both $K_{1,4}$ and $K_{5}$. Clearly, $\tau$ is not realizable in $K_{1,4}$ under $L$ since $\left(\{c\},\{b\}, V\left(K_{1,4}\right)-\{c, b\}\right)$ is not a correct realization of $\tau$ in $K_{1,4}$. However, $\tau$ is realizable in $K_{5}$ under $L$ since $\left(\{a\},\{b\}, V\left(K_{5}\right)-\{a, b\}\right),\left(\{a\},\{e\}, V\left(K_{5}\right)-\right.$ $\{a, e\}),\left(\{c\},\{b\}, V\left(K_{5}\right)-\{c, b\}\right)$ and $\left(\{c\},\{e\}, V\left(K_{5}\right)-\{c, e\}\right)$ are correct realizations of $\tau$ in $K_{5}$.

We now investigate the computational complexity of the problem associated with the definition above.

Dynamic Realizable Sequence - DynRealSeq

Instance: A graph $G$, a sequence $\tau=\left(n_{1}, \ldots, n_{p^{\prime}}, \ldots, n_{p}\right)$ with size $p \geq p^{\prime}$, and a $\left(n_{1}, \ldots, n_{p^{\prime}}\right)$-partition-hierarchy $L$ for $\tau$ and $G$.

Question: Is $\tau$ realizable in $G$ under $L$ ?

As understood above, DynRealSEQ is a $\Pi_{2}^{p}$-complete problem. Our proof of this claim is based on our reduction from 1-IN-3 SAT to REALSEQ (Section 2). In order to reuse it, we need a $\Pi_{2}^{p}$-complete version of 1-IN-3 SAT.

$\forall \exists 1$-IN-3 SAT

Instance: A $3 \mathrm{CNF}$ formula $F$ over variables $X \cup Y$ and clauses $\left\{C_{1}, \ldots, C_{m}\right\}$, where $X=\left\{x_{1}, \ldots, x_{n^{\prime}}\right\}, Y=\left\{x_{n^{\prime}+1}, \ldots, x_{n}\right\}$ and $n^{\prime} \leq n$.

Question: For every truth assignment of the variables of $X$, does there exist a truth assignment of the variables of $Y$ such that $F$ is satisfied in a 1-in-3 way?

We first show below that $\forall \exists 1$-IN-3 SAT is $\Pi_{2}^{p}$-complete by reduction from the following classical $\Pi_{2}^{p}$-complete problem.

$\forall \exists 3 \mathrm{SAT}$

Instance: A $3 \mathrm{CNF}$ formula $F$ over variables $X \cup Y$, where $X=\left\{x_{1}, \ldots, x_{n^{\prime}}\right\}$, $Y=\left\{x_{n^{\prime}+1}, \ldots, x_{n}\right\}$ and $n^{\prime} \leq n$, and clauses $\left\{C_{1}, \ldots, C_{m}\right\}$.

Question: For every truth assignment of the variables of $X$, does there exist a truth assignment of the variables of $Y$ such that $F$ is satisfied?

Lemma $6 \forall \exists 1$-IN-3 SAT is $\Pi_{2}^{p}$-complete.

Proof $\forall \exists 1$-IN-3 SAT is clearly in $\Pi_{2}^{p}$. One can indeed design an algorithm that takes $F$ and a truth assignment $\phi_{1}$ to the variables of $X$ for which there is no truth assignment $\phi_{2}$ to the variables in $Y$ making $F$ evaluated in a 1-in-3 way as input. It just has to check that $\phi_{2}$ does not exist thanks to an oracle dealing with 1-IN-3 SAT. Such a checking algorithm runs in polynomial time regarding the size of $F$.

We now show that $\forall \exists 1$-IN-3 SAT is $\Pi_{2}^{p}$-complete by reduction from $\forall \exists 3 \mathrm{SAT}$. From a $3 \mathrm{CNF}$ formula $F$ over variables $X \cup Y$, we construct a new $3 \mathrm{CNF}$ formula $F^{\prime}$ over variables $X^{\prime} \cup Y^{\prime}$ such that for every truth assignment $\phi_{1}$ to the variables in $X$ there exists a truth assignment $\phi_{2}$ to the variables in $Y$ making $F$ evaluated true if and only if for every truth assignment $\phi_{1}^{\prime}$ to the variables 


\begin{tabular}{|c|c|c|c|c|c|c|}
\hline$\left(\phi_{3}\left(\ell_{i_{1}}\right), \phi_{3}\left(\ell_{i_{2}}\right), \phi_{3}\left(\ell_{i_{3}}\right)\right)$ & $\phi_{2}^{\prime}\left(a_{i}\right)$ & $\phi_{2}^{\prime}\left(b_{i}\right)$ & $\phi_{2}^{\prime}\left(c_{i}\right)$ & $\phi_{2}^{\prime}\left(d_{i}\right)$ & $\phi_{2}^{\prime}\left(e_{i}\right)$ & $\phi_{2}^{\prime}\left(f_{i}\right)$ \\
\hline$(1,0,0)$ & 1 & 0 & 0 & 0 & 0 & 0 \\
\hline$(0,1,0)$ & 0 & 0 & 1 & 0 & 0 & 0 \\
\hline$(0,0,1)$ & 0 & 0 & 0 & 0 & 1 & 0 \\
\hline$(1,1,0)$ & 1 & 0 & 0 & 1 & 0 & 0 \\
\hline$(1,0,1)$ & 1 & 0 & 0 & 0 & 0 & 1 \\
\hline$(0,1,1)$ & 0 & 0 & 1 & 0 & 0 & 1 \\
\hline$(1,1,1)$ & 1 & 0 & 0 & 1 & 0 & 1 \\
\hline
\end{tabular}

Table 1 Truth assignment of $\phi_{2}^{\prime}$ for the variables in $Y-Y^{\prime}$

in $X^{\prime}$ there exists a truth assignment $\phi_{2}^{\prime}$ to the variables in $Y^{\prime}$ such that $F^{\prime}$ is evaluated true in a 1 -in-3 way.

The reduction is straightforward. First, replace each clause $C_{i}=\left(\ell_{i_{1}} \vee\right.$ $\left.\ell_{i_{2}} \vee \ell_{i_{3}}\right)$ in $F$ by four clauses $\left(\overline{\ell_{i_{1}}} \vee a_{i} \vee b_{i}\right),\left(\overline{\ell_{i_{2}}} \vee c_{i} \vee d_{i}\right),\left(\overline{\ell_{i_{3}}} \vee e_{i} \vee f_{i}\right)$ and $\left(a_{i} \vee c_{i} \vee e_{i}\right)$ in $F^{\prime}$ where $a_{i}, b_{i}, c_{i}, d_{i}, e_{i}$ and $f_{i}$ are six new variables associated with $C_{i}$. Finally, let $X^{\prime}=X$ and $Y^{\prime}=Y \cup \bigcup_{i=1}^{m}\left\{a_{i}, b_{i}, c_{i}, d_{i}, e_{i}, f_{i}\right\}$. Note that $F^{\prime}$ has $4 m$ clauses and may be obtained easily.

First suppose that for every truth assignment $\phi_{1}^{\prime}$ to the variables of $X^{\prime}$ there exists a truth assignment $\phi_{2}^{\prime}$ to the variables in $Y^{\prime}$ such that $F^{\prime}$ is satisfied in a 1-in-3 way. Because every clause of $F^{\prime}$ has exactly one true literal under $\phi_{1}^{\prime}$ and $\phi_{2}^{\prime}$, it means that only one element in $\left\{a_{i}, c_{i}, e_{i}\right\}$ is evaluated true by $\phi_{2}^{\prime}$ for every $i \in\{1, \ldots, m\}$. Let us suppose that for such an $i$ we have $\phi_{2}^{\prime}\left(a_{i}\right)=1$ and $\phi_{2}^{\prime}\left(c_{i}\right)=\phi_{2}^{\prime}\left(e_{i}\right)=0$ without loss of generality. Thus, we have $\ell_{i_{1}}$ evaluated true by either $\phi_{1}^{\prime}$ or $\phi_{2}^{\prime}$. It follows that the following truth assignment $\phi_{1}$ and $\phi_{2}$ of the variables in $X$ and $Y$, respectively,

$-\phi_{1}=\phi_{1}^{\prime}$,

- $\phi_{2}(x)=\phi_{2}^{\prime}(x)$ for every $x \in Y$,

is such that $F$ is satisfied. Conversely, suppose that for every truth assignment $\phi_{1}$ to the variables in $X$ there is a truth assignment $\phi_{2}$ to the variables in $Y$ such that $F$ has all its clauses satisfied under $\phi_{1}$ and $\phi_{2}$. We explain how to get a truth assignment $\phi_{2}^{\prime}$ to the variables in $Y^{\prime}$ so that $F^{\prime}$ is evaluated true in a 1-in-3 way under $\phi_{2}^{\prime}$ and the truth assignment $\phi_{1}^{\prime}=\phi_{1}$ to the variables in $X^{\prime}$. First, let $\phi_{2}^{\prime}(x)=\phi_{2}(x)$ for every $x \in Y$. We then have to provide a truth assignment of $a_{i}, b_{i}, c_{i}, d_{i}, e_{i}$ and $f_{i}$ via $\phi_{2}^{\prime}$ for every $i \in\{1, \ldots, m\}$. This assignment depends on the number of true literals in $C_{i}=\left(\ell_{i_{1}} \vee \ell_{i_{2}} \vee \ell_{i_{3}}\right)$ via $\phi_{1}$ and $\phi_{2}$. Let $\phi_{3}: X \cup Y \rightarrow\{0,1\}$ be the truth assignment of the variables in $X \cup Y$ deduced from $\phi_{1}$ and $\phi_{2}$ as follows:

- if $i \in\left\{1, \ldots, n^{\prime}\right\}$, then $\phi_{3}\left(x_{i}\right)=\phi_{1}\left(x_{i}\right)$;

- if $i \in\left\{n^{\prime}+1, \ldots, n\right\}$, then $\phi_{3}\left(x_{i}\right)=\phi_{2}\left(x_{i}\right)$.

Consider now that the images of the $a_{i}$ 's, $b_{i}$ 's, $c_{i}$ 's, $d_{i}$ 's, $e_{i}$ 's and $f_{i}$ 's by $\phi_{2}^{\prime}$ are the ones depicted in Table 1 . It should then be clear that $F^{\prime}$ is evaluated true in a 1-in-3 way under $\phi_{1}^{\prime}$ and $\phi_{2}^{\prime}$. 
We finally prove that DyNREALSEQ is $\Pi_{2}^{p}$-complete.

Theorem 7 DynRealSeq is $\Pi_{2}^{p}$-complete.

Proof DynReALSEQ is clearly a $\Pi_{2}^{p}$ problem. One can provide a combination of parts $\left(V_{1}, \ldots, V_{p^{\prime}}\right)$ from the $\left(n_{1}, \ldots, n_{p^{\prime}}\right)$-partition-hierarchy for $\tau$ and $G$ of the problem instance to a polynomial-time algorithm checking that these parts cannot be extended to a realization of $\tau$ in $G$. It just has to make sure that $\tau$ is admissible for $G$, and the sequence $\left(n_{p^{\prime}+1}, \ldots, n_{p}\right)$ is not realizable in $G-$ $\bigcup_{i=1}^{p^{\prime}} V_{i}$ using an oracle for REALSEQ. Note further that DyNREALSEQ can be neither in co-NP (for the same reason as REALSEQ is not in co-NP) nor in NP since the number of partial realizations encoded by the partition-hierarchy may be exponential regarding the input size.

We now show that DyNREALSEQ is complete in $\Pi_{2}^{p}$ by reduction from $\forall \exists 1$-IN-3 SAT ( $\Pi_{2}^{p}$-complete by Lemma 6 ). Our reduction is inspired by the reduction from 1-IN-3 SAT to REALSEQ we gave in the proof of Theorem 1. Remember that in this reduction, setting a variable of $F$ to true is simulated in an instance of REALSEQ by adding a literal vertex of $G_{F}$ to the part with size $n_{1}+n$ of a realization of $\tau_{F}$ in $G_{F}$. We here somehow want to keep that relationship between setting a variable of $F$ to true and putting a literal vertex of the $G_{F}$ into a part of the realization. Given a truth assignment $\phi_{1}$ to the variables in $X$, it means that we have to check whether every partial realization of $\tau_{F}$ in $G_{F}$ whose part with size $n_{1}+n$ contains the literal vertices associated with the true literals via $\phi_{1}$ is extendible to a realization of $\tau_{F}$ in $G_{F}$. All these possible partial realizations are considered using a partition-hierarchy for $\tau_{F}$ and $G_{F}$.

First of all, let $G_{F}$ be the graph obtained from $F$ using the reduction we gave in the proof of Theorem 1 . Then, let $\tau_{F}=\left(1, \ldots, 1, n_{1}+n-n^{\prime}, n_{2}-n\right)$ be a sequence with size $n^{\prime}+2$ admissible for $G_{F}$, let $L_{i}=\left\{\left\{v_{x_{i}}\right\},\left\{v_{\overline{x_{i}}}\right\}\right\}$ be a 1-partition-level for $\tau_{F}$ and $G_{F}$ for every $x_{i} \in X$, and $L=\bigcup_{i=1}^{n^{\prime}} L_{i}$ be a $(1, \ldots, 1)$-partition-hierarchy for $\tau_{F}$ and $G_{F}$. With a truth assignment $\phi_{1}$ of the variables in $X$ setting $n^{\prime}$ literals of $F$ to true is then associated a combination of vertex-disjoint subsets $\left(V_{1}, \ldots, V_{n^{\prime}}\right)$ from the 1-partition-levels in $L$, where $V_{i}=\left\{x_{i}\right\}$ if $\phi_{1}\left(x_{i}\right)=1$ or $V_{i}=\left\{\overline{x_{i}}\right\}$ otherwise.

Let us now suppose that for every truth assignment $\phi_{1}$ to the variables in $X$ there exists a truth assignment $\phi_{2}$ to the variables of $Y$ such that $F$ is evaluated true in a 1 -in-3 way. Then the realization $\left(V_{1}, \ldots, V_{n^{\prime}+2}\right)$ of $\tau_{F}$ in $G_{F}$, where

- for every $i \in\left\{1, \ldots, n^{\prime}\right\}$, we have $V_{i}=\left\{v_{x_{i}}\right\}$ if $\phi_{1}\left(x_{i}\right)=1$ or $V_{i}=\left\{v_{\overline{x_{i}}}\right\}$ otherwise,

- $V_{n^{\prime}+1}$ contains all the vertices from the base subgraph of $G_{F}$ and every literal vertex $v_{\ell_{i}}$ of the clause subgraph of $G_{F}$ such that $\phi_{2}\left(\ell_{i}\right)=1$,

$-V_{n^{\prime}+2}=V\left(G_{F}\right)-\bigcup_{i=1}^{n^{\prime}+1} V_{i}$,

is correct according to the arguments we gave in the proof of Theorem 1 . Conversely, suppose that every combination $\left(V_{1}, \ldots, V_{n^{\prime}}\right)$ of subsets from the 
1-partition-levels of $L$ is extendable to a realization $\left(V_{1}, \ldots, V_{n^{\prime}+2}\right)$ of $\tau_{F}$ in $G_{F}$. As explained before, the partition $\left(V_{1}, \ldots, V_{n^{\prime}}\right)$ is associated with a truth assignment $\phi_{1}$ to the variables in $X$, and from the literal vertices contained in $V_{n^{\prime}+1}$ we can deduce a truth assignment $\phi_{2}$ to the variables in $Y$ (see the proof of Theorem 1). Clearly, $F$ is evaluated true in a 1-in-3 way under $\phi_{1}$ and $\phi_{2}$.

\section{References}

1. D. Barth, O. Baudon, and J. Puech. Decomposable trees: a polynomial algorithm for tripodes. Discret. Appl. Math., 119(3):205-216, July 2002.

2. D. Barth and H. Fournier. A degree bound on decomposable trees. Discret. Math., 306(5):469-477, 2006.

3. O. Baudon, J. Bensmail, J. Przybyło, and M. Woźniak. Partitioning powers of traceable or Hamiltonian graphs. Preprint, 2012. Available at http://hal.archives-ouvertes.fr/hal00687278.

4. M.E. Dyer and A.M. Frieze. On the complexity of partitioning graphs into connected subgraphs. Discret. Appl. Math., 10:139-153, 1985.

5. N. Guttmann-Beck and R. Hassin. Approximation algorithms for min-max tree partition. J. Algorithms., 24(2):266-286, 1997.

6. E. Györi. On division of graphs to connected subgraphs. In Combinatorics, pages 485494, Colloq. Math. Soc. János Bolyai 18, 1978.

7. L. Lovász. A homology theory for spanning trees of a graph. Acta Math. Acad. Sci. Hung., 30(3-4):241-251, 1977. 